\title{
A cluster of high psychological and somatic symptoms in children with idiopathic scoliosis predicts persistent pain and analgesic use 1 year after spine fusion
}

\author{
Terri Voepel-Lewis $^{1}$ (D) | Michelle S. Caird ${ }^{2}$ | Alan R. Tait $^{1}$ | Frances A. Farley ${ }^{2}$ | \\ Ying Li $^{2}$ | Shobha Malviya ${ }^{1}$ | Afton Hassett ${ }^{1}$ | Monica Weber ${ }^{1}$ | Emily Currier ${ }^{1}$ | \\ Trevor de Sibour $^{1}$ | Daniel J. Clauw ${ }^{1}$
}

${ }^{1}$ Department of Anesthesiology, University of Michigan, Ann Arbor, Michigan

${ }^{2}$ Department of Orthopedic Surgery, University of Michigan, Ann Arbor, Michigan

\section{Correspondence}

Terri Voepel-Lewis, Department of Anesthesiology, Room 4917 Children's and Women's Hospital, University of Michigan, http://www.med.umich.edu, $1540 \mathrm{E}$.

Hospital Drive, Ann Arbor, MI 48109-4245.

Email: terriv@umich.edu

Funding information

The study was funded by departmental resources only.

Section Editor: Dr Suellen Walker

\section{Summary \\ Background: Persistent postoperative pain is a significant problem for many chil- dren, particularly for those undergoing major surgery such as posterior spine fusion. More than two-thirds report persistent pain after spine fusion, yet factors that may contribute to poorer outcomes remain poorly understood.}

Aims: This prospective, longitudinal study examined how psychologic and somatic symptoms cluster together in children aged 10-17 years with idiopathic scoliosis, and tested the hypothesis that a higher psychological and somatic symptom cluster would predict worse pain outcomes 1 year after fusion.

Methods: Otherwise healthy children with idiopathic scoliosis completed preoperative surveys measuring recent pain intensity, pain location(s), somatic symptom severity, painDETECT (neuropathic-type pain symptoms), pain interference, fatigue, depression, anxiety, and pain catastrophizing. Pain outcome data were collected during hospitalization, and at 1 year after surgery.

Results: Ninety-five children completed baseline surveys and a cluster analysis differentiated 28 (30\%) with a high symptom profile that included; higher depression, fatigue, pain interference, catastrophizing, and painDETECT scores. High symptom cluster membership independently predicted higher pain interference at 1 year ( $\beta 9.92$ [95\% Cl 6.63, 13.2], $P<0.001$ ). Furthermore, children in this high symptom cluster reported significantly higher pain intensity and painDETECT scores, and had a $50 \%$ higher probability of continued analgesic use at 1 year compared to those in the Low Symptom Cluster (95\% Cl 21.3-78.5, $P=0.001)$.

Conclusion: Findings from this exploratory study suggest a need to comprehensively assess children with scoliosis for preoperative signs and symptoms that may indicate an underlying vulnerability for persistent pain. This, in turn may help guide a comprehensive perioperative treatment strategy to mitigate the potential for longterm pain trajectories.

KEYWORDS

adolescents, children, chronic pain, musculoskeletal pain, pediatric pain, postoperative pain 


\section{1 | INTRODUCTION}

Persistent postoperative pain poses a significant healthcare problem for many children and adolescents. Of children, 13-22\% report persistent pain 6-12 months after major surgery, ${ }^{1-3}$ with one-third reporting daily pain and $14 \%$, severe pain. ${ }^{2}$ Lower rates (5\%) are reported following less invasive outpatient procedures, ${ }^{4}$ while highest rates (up to 75\%) are found following posterior spine fusion. ${ }^{5,6}$ Longitudinal studies have described moderate to severe pain in nearly one-third of children with idiopathic scoliosis that remained unimproved or worsened for $16 \%$ for up to 5 years after surgical correction. ${ }^{5,7,8}$ Importantly, no obvious nociceptive source for pain could be identified in $85 \%$ of children with persistent pain after fusion. ${ }^{9}$ Furthermore, the degree and location of curve, surgical approach, fusion length, and magnitudes of correction correlate poorly or not at all with persistent pain, and removal of hardware after 2 years did not relieve pain for $40 \%$ of children with severe pain. ${ }^{5,7,8,10}$ Such data suggest that nonnociceptive factors may better explain persistent pain after surgery.

Despite the high prevalence and potential negative impact of persistent postoperative pain in children, risk factors remain poorly understood. Several studies in small samples of children suggest that child parental psychosocial factors help to explain persistent pain after major surgery. ${ }^{2,11}$ However, as summarized in a recent systematic review, variations in study methods and sample characteristics limit the interpretation of data and call for further study. ${ }^{3}$ On the other hand, this growing body of evidence suggests that a significant portion of children with scoliosis exhibit characteristics prior to surgery that indicate a particular vulnerability for a long-term pain trajectory. Such factors include, pain presence and severity, anxiety sensitivity, poorer coping, negative mood, higher analgesic use, and simple markers of functioning such as missed school days. ${ }^{5-7,9}$ We recently described a cluster of self-reported psychological and somatic symptoms that was present in $30 \%$ of those with idiopathic scoliosis prior to surgery. ${ }^{12}$ These symptoms included higher depression, fatigue, multisite pain and somatic symptomology, neuropathic pain symptoms, pain interference, and pain catastrophizing. Furthermore, children with this cluster of symptoms experienced higher pain and analgesic use in the immediate 2 weeks following surgery. We postulated that this symptom cluster may be related to the same phenomenon commonly seen in subsets of adults with chronic pain and indicative of greater central nervous system contributions to pain (ie, central pain sensitization or augmentation). ${ }^{13}$ These and other investigators have argued that the phenotype associated with chronic postoperative pain is similar to that found in patients with centralized pain sensitization which, in turn, is supported by demonstrated differences in quantitative sensory testing and neuroimaging. ${ }^{13}$

The present study continued our earlier exploratory work with the aim to determine whether children who self-report a cluster of higher psychological and somatic symptoms would remain vulnerable to persistent postoperative pain 1 year following posterior spine fusion. Specifically, we tested the following hypotheses: (a) Children with higher psychological and somatic symptomology prior to

\section{What is already known}

- Persistent postoperative pain occurs in one of five children undergoing major surgery, yet factors associated with this are uncertain.

\section{What this paper adds}

- Children exhibiting a cluster of psychological and somatic symptoms, suggesting a pain vulnerable trait preoperatively, report worse pain outcomes 1 year after spine fusion.

surgery would report higher pain and pain interference 1 year after posterior spine fusion; (b) These high risk children would also be more likely to need ongoing analgesic use at 1 year.

\section{MATERIALS AND METHODS}

With approval from the institutional review board at the University of Michigan and written parental consent and child assent, we consecutively recruited cognitively intact, English-speaking children aged 10-17 years scheduled to undergo primary elective posterior spine fusion for surgical correction of idiopathic scoliosis from July 2014 to December 2016. We excluded those who had previous spine surgery and those with cognitive impairments who could not independently complete surveys. One-year follow-up concluded in January 2018. The study design was a prospective, longitudinal observational study.

\section{1 | Measures}

Pain Intensity was self-reported by participants with the 0-10 numeric rating scale (NRS) where $0=$ no pain and $10=$ "worst pain possible". ${ }^{14}$

Pediatric Fibromyalgia Survey Criteria ( $\mathrm{pFSC}$ ) combines scores from the two-sided body map (ie, child's self-reported pain locations) and a modified Somatic Symptom Severity tool (child's global perceptions of somatic symptoms) to identify widespread pain and symptoms common in children and adults with centralized pain conditions such as fibromyalgia (score range 0-31). ${ }^{15,16} \mathrm{~A}$ similar version of the fibromyalgia survey criteria was recently found to have excellent sensitivity and specificity for diagnosing juvenile fibromyalgia in adolescents. ${ }^{16}$ Scores from our pFSC were found to be reliable in this age group (ICC 0.912 [95\% $\mathrm{Cl} 0.845-0.95]$ ) and helped to differentiate symptom clusters in our previous work. ${ }^{12}$ Scores range from 0 to 31 and are treated as continuous to reflect what is now considered to be a continuum of pain centralization. ${ }^{17}$

Widespread pain was defined as pain in three or more regions of the body as identified on the self-reported body map and as defined previously. ${ }^{18}$ 
painDETECT documented the presence and extent of neuropathic-type pain symptoms with scores ranging from 0 to 39 . We previously demonstrated that this measure has good internal consistency (Cronbach $\alpha 0.757$ [95\% Cl 0.662-0.834]) and "test-retest" reliability (ICC 0.77 [95\% Cl 0.365-0.811]) in this age group. ${ }^{12}$

Pediatric Patient-Reported Outcome Measurement System (PROMIS) Short Forms were used to measure somatic and psychologic symptoms including, fatigue, depression, anxiety, and pain interference. These measures have been found to have good reliability in children and yield scores ranging from 0 to 32 (pain interference, depression, anxiety) or 0-40 (fatigue). ${ }^{19,20}$

Pain Catastrophizing measures the degree of "pain worry" in children. Scores range from 0 to 52 , and the instrument has excellent psychometric properties and predictive validity. ${ }^{21}$

\section{2 | Procedures}

Following parental consent and child assent in the preoperative clinic, children independently completed the baseline surveys using an iPad with a Qualtrics link. The surveys included automatic reminder messages for missing items in order to encourage complete responses and reduce missing data. The survey contained only a unique identifier to ensure privacy and honest disclosure. Approximately 2 weeks after the clinic visit, children underwent posterior spine fusion and we recorded the child's demographics, surgical information, and all analgesics administered (opioids were converted to oral morphine equivalents per kilogram per hour of hospital stay). Children were visited each day in the hospital to obtain self-reported pain intensity scores. One year after surgery, children completed a paper follow-up survey (in person at the follow-up clinic visit or by mail) regarding recent and current pain presence, intensity, pain interference, neuropathic pain symptoms, and analgesic use.

\section{3 | Statistical analyses}

Missing data were minimal $(<3$ items for the baseline depression survey in two children). These missing items were imputed from the average of the completed depression items. We used SPSS (version 24) to analyze all data. Pain measures and surveys were considered continuous measures and summarized with descriptive statistics as means \pm standard deviations (SD), and medians (M) with percentiles, where applicable and based on tests of normality. Nominal data are reported as $\mathrm{n}$ (\%).

We first conducted a series of two-step cluster analyses which uses a log-likelihood distance measure to differentiate or profile children based on the ideal number of homogeneous structures suggested by the data, and not defined a priori. The two-step is robust to violations in assumptions of independence and normality, and, for these analyses, the Akaike information criterion was chosen as the clustering criterion. Our models included all of the baseline symptom variables of interest (ie, PROMIS depression, anxiety, and pain interference, painDETECT, pain catastrophizing, and pFSC) since these were deemed to measure relevant and nonredundant attributes (ie, all correlations $<0.76^{22}$ ). We subsequently removed the noncontributing factors (ie, nonsignificant or $P<0.05$ ) anxiety and $\mathrm{pFSC}$, until a final solution with a high measure of cohesion was obtained. The resultant cluster groups of children were compared with univariate analyses (chi square, unpaired t or Mann-Whitney $U$ tests) with regard to demographics, preoperative factors, surgical course, and 1year pain outcomes. Mean differences (MD) or odd ratios (OR) with 95\% confidence intervals $(\mathrm{Cl})$ are reported. We then tested the hypotheses using multivariable linear regression for the outcome, Pain Interference, and a mixed effect logistic regression for the outcome, ongoing analgesic use. The following covariates were included in the models together with the primary factor of interest, cluster membership: age, sex, body mass index, spine levels fused, intraoperative analgesic technique (ie, intrathecal vs other), average inhospital NRS score, and total morphine consumption.

We recruited 100 children to obtain a sample large enough to estimate a $20 \%$ proportion with multisite pain and symptomology (95\% $\mathrm{Cl}=0.20)$, allowing for an estimated $25 \%$ loss-to-follow-up. The resultant sample $(n=75)$ was determined, a priori, to be more than sufficient to include up to 6 variables in a two-step cluster analysis (recommended $2^{\mathrm{k}}$ ), ${ }^{23}$ and to detect at least a $40 \%$ difference in analgesic requirement (ie, the smallest expected effect size) at 1 year $(\alpha=0.05 ; \beta=0.20)$.

\section{3 | RESULTS}

One hundred and twenty-seven children were approached for this study, and 23 declined participation or did not meet inclusion criteria. One hundred and four children were enrolled; however, we excluded three due to surgical variation or secondary spine fusion, two who could not independently complete baseline surveys, and four who had surgery postponed or canceled. Ninety-five children completed all baseline surveys and underwent surgery. The final cluster analyses differentiated two groups with a high degree of separation and cohesion. Figure 1 depicts, in order of contribution, the psychological and somatic symptom scores that best differentiated a High Symptom Cluster of children (prevalence $=28$ [95\% Cl 22-38]) from a Low Symptom Cluster of children. Table 1 describes the baseline characteristics and the surgical/hospital course of children in these clusters. In addition to the cluster factor differences, children in the High Symptom Cluster reported higher preoperative pain intensity, anxiety, and pFSC scores were more likely to report widespread pain and to have been using analgesics compared with children in the Low Symptom Cluster. These children also reported higher pain intensity during their hospital stay, but used similar amounts and types of analgesics postoperatively.

Seventy-six children ( $80 \%$ of those recruited) completed all parts of the 1-year follow-up survey, and nearly all of these did so in person at their 1-year clinic visit. The $20 \%$ lost to follow-up were excluded from the longitudinal analyses. The group lost to follow-up, however, was not different from children who completed the 1 year follow-up with regard to female sex $(68 \%$ vs $78 \%, P=0.402)$, age 


\section{Description of Clusters}

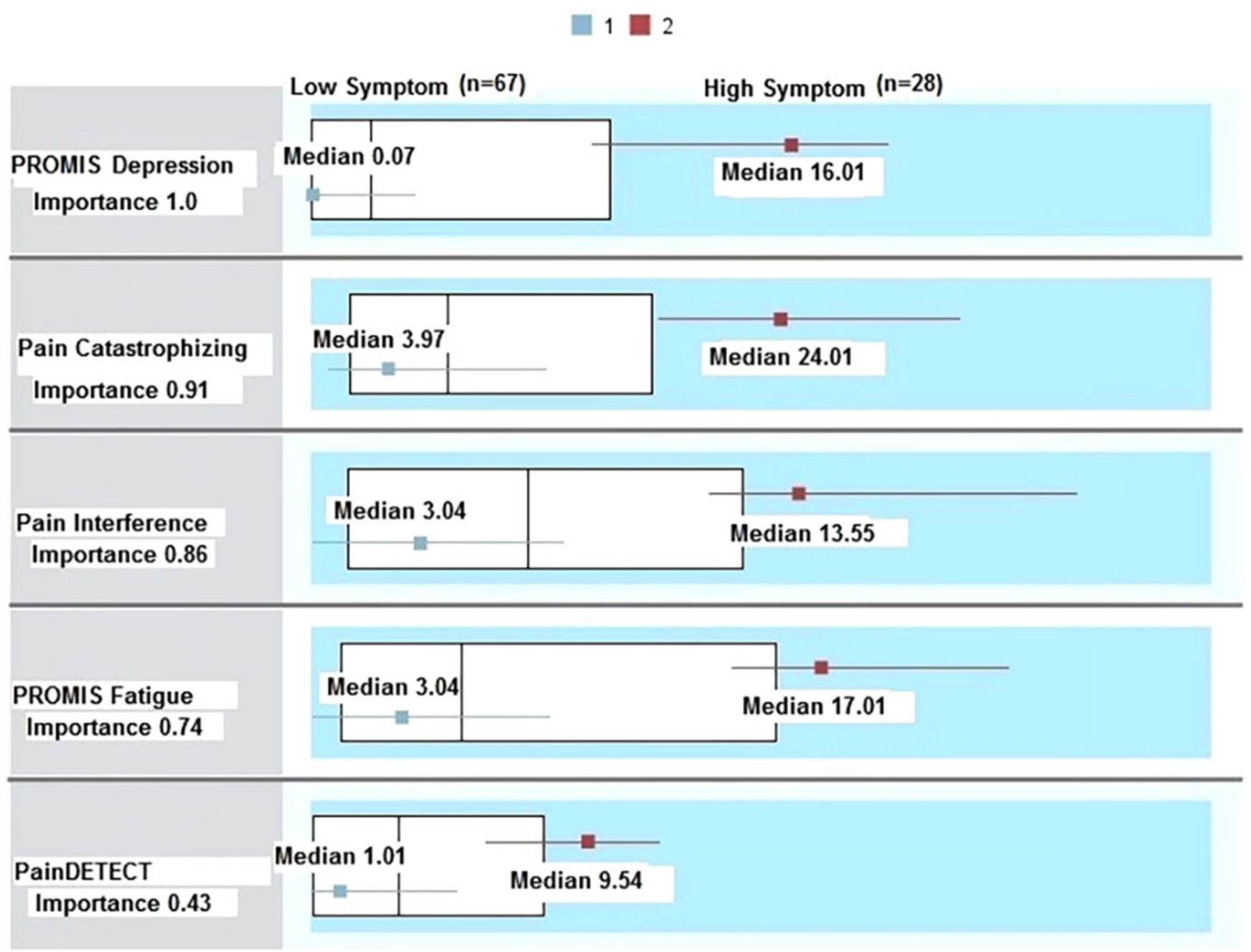

FIGURE 1 Description of Factors in Order of their Contribution to Low and High Symptom Cluster Membership. Results of the two-step cluster analysis; Silhouette measure of cohesion and separation $>0.5$ (good); Boxes depict the median (line) and 25th to 75 th percentile range for the entire sample. The blue and red horizontal lines depict the interquartile range for the Low and High Symptom Cluster Groups, respectively [Colour figure can be viewed at wileyonlinelibrary.com]

(14.5 vs 13.9 years, $P=0.255$ ), body mass index (23.1 vs $21.7 \mathrm{~kg} / \mathrm{m}^{2}$, $P=0.301)$, baseline scores on catastrophizing (11.9 vs 11.6, $P=0.907$ ), PROMIS depression (8.3 vs $5.5, P=0.158$ ), fatigue (10.1 vs $8.2, P=0.385$ ), anxiety (10.6 vs $11.7, P=0.646$ ), painDETECT (4.3 vs $4.9, P=0.668$ ), or baseline presence of widespread pain (26\% vs $29 \%, P=0.820$ ).

The High Symptom Cluster reported significantly higher pain intensity, pain interference, and neuropathic-type pain and were more likely to be taking analgesics (primarily over-the-counter nonopioids) and using nonpharmacologic pain (primarily acupuncture [20\%] and meditation [17\%]) treatments at 1 year (see Table 2). Additionally, the pain outcomes were highly correlated with rho coefficients ranging from 0.598 to $0.785, P<0.01$. When adjusted for other baseline characteristics and hospital course variables, Cluster Membership independently predicted pain interference scores ( $\beta=9.92$ [95\% Cl 6.63, 13.2], $P<0.001$; see Table 3). The mixed effect logistic regression model demonstrated that, adjusted for mean values of all baseline characteristics and hospital course variables, children in the High Symptom Cluster had a 50\% higher probability of continued analgesic use at 1 year compared to those in the Low Symptom Cluster (estimated marginal mean $=50 \%$ [95\% Confidence Interval $=21.3,78.5], P=0.001$ ) .

Of note, while children in the High Symptom Cluster had higher ongoing pain and pain interference at 1 year, they did experience a significant decrease in reported PROMIS pain interference from their baseline scores ( $\mathrm{MD}-5.65$ [95\% $\mathrm{Cl} 2.6$, 8.7] paired $t P=0.001$ ). In contrast, pain interference scores in the Low Symptom Cluster were no different from baseline (MD $0.85[95 \% \mathrm{Cl}-0.47,2.18] P=0.20$ ).

\section{DISCUSSION}

Findings from this longitudinal study suggest that $30 \%$ of children with idiopathic scoliosis can be differentiated by a high degree of 
TABLE 1 Baseline and surgical course characteristics of the cluster profile groups

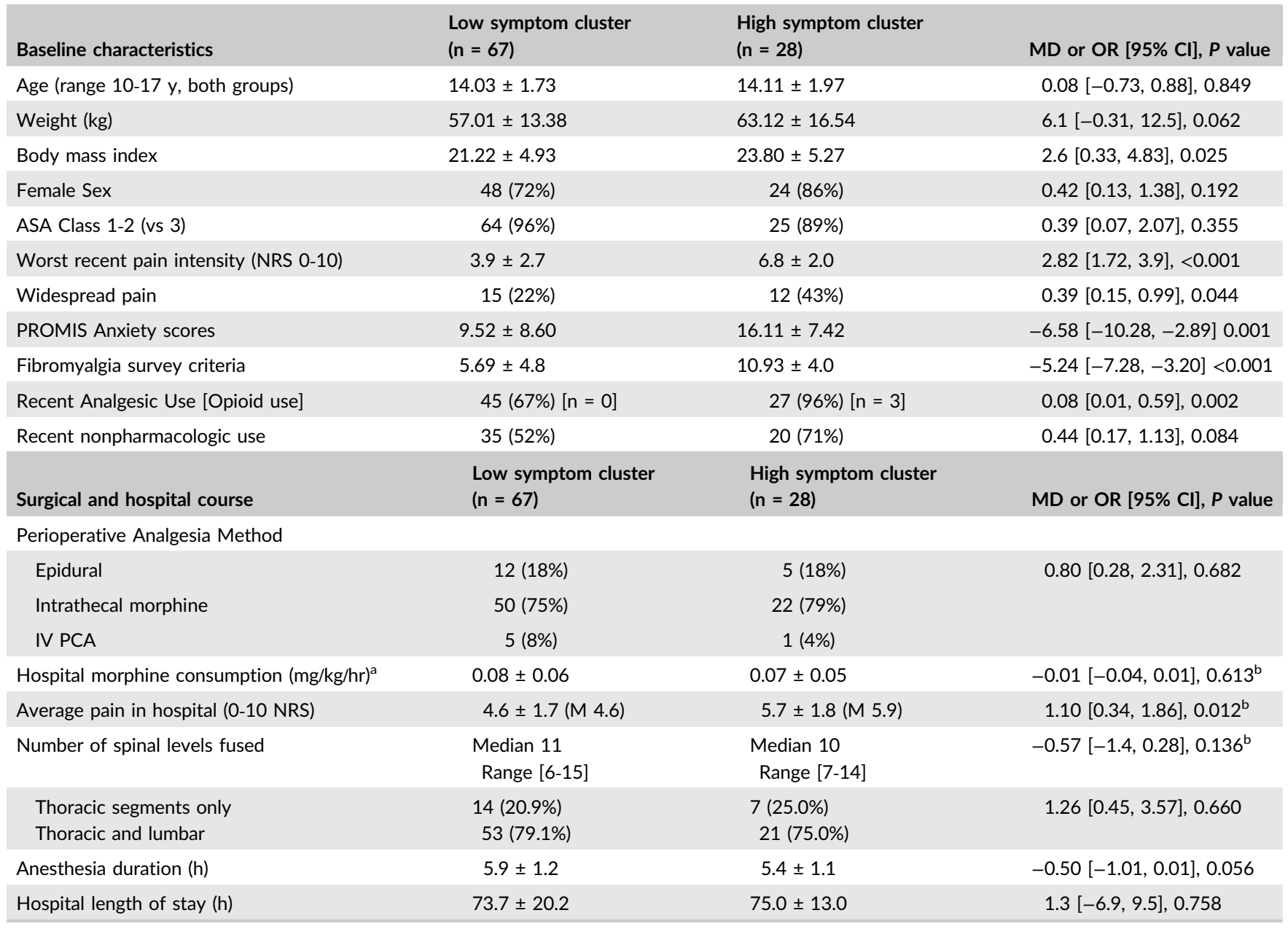

Data are presented as mean \pm standard deviation or as $\mathrm{n}(\%)$, where applicable.

Cl, Confidence Interval; IVPCA, Intravenous Patient Controlled Analgesia; M, Median; MD, Mean Difference; NRS, Numeric Rating Scale; OR, Odds Ratio.

${ }^{a}$ All opioids converted to morphine equivalents for this variable.

${ }^{b}$ Mann-Whitney $U$ test of significance for nonparametric data.

psychological and somatic symptomology prior to surgery including; higher depression, pain interference, pain catastrophizing, fatigue, and neuropathic-type pain symptoms. Children with this cluster of symptoms reported significantly greater pain interference and were $50 \%$ more likely to be taking analgesics (primarily nonopioids) 1 year after posterior spine fusion. These data suggest that, even before surgery, a pain vulnerable profile that predicts the observed longterm postoperative trajectory can be identified in children with scoliosis who require posterior spine fusion.

Importantly, children in the Low and High Symptom Cluster groups did not differ with regard to surgical characteristics, perioperative, and hospital management, and these factors were not independently associated with long-term outcomes. We did find, however, that the High Symptom Cluster reported higher pain intensity after surgery and at 1 year. Unlike previous data, ${ }^{2}$ we and others ${ }^{4}$ did not find an association between postoperative pain intensity scores and persistent pain. This discrepancy in findings may be due to the presence and stronger predictive value of the baseline cluster of psychological and somatic symptoms (ie, depression, fatigue, pain worry, neuropathic-like symptoms). Others have similarly found strong and independent associations between psychological characteristics such as "anxiety sensitivity", 2,24 child pain catastrophizing ${ }^{25}$ and long-term postoperative pain outcomes. Our data provide a more comprehensive description of the psychological and somatic characteristics that may cluster together to predict long-term pain trajectories after surgery. As such, findings suggest a need to characterize symptomology in children with idiopathic scoliosis before surgery, and to initiate symptom reduction strategies before and immediately after posterior spine fusion. Targeted interventions might include psychologic, nonpharmacologic, and possibly pharmacologic strategies to reduce depression symptoms, promote sleep and coping capacity, and relieve symptoms of neuropathic-type of pain.

Emerging evidence of multisite and symptom clusters across differing chronic and recurrent pain syndromes in children suggest that they may not be separate disorders, but rather early manifestations of an underlying pain phenotype that persists into adulthood. 


\begin{tabular}{|c|c|c|c|}
\hline & $\begin{array}{l}\text { Low symptom } \\
\text { cluster }(n=56)\end{array}$ & $\begin{array}{l}\text { High symptom } \\
\text { cluster }(n=20)\end{array}$ & $\begin{array}{l}\text { MD or } \mathrm{OR}[95 \% \mathrm{Cl}] \\
P \text { value }\end{array}$ \\
\hline $\begin{array}{l}\text { Recent high pain score } \\
\text { (range 0-10) }\end{array}$ & $\begin{aligned} 2.07 & \pm 2.0 \\
M & =1\end{aligned}$ & $\begin{aligned} 4.8 & \pm 2.5 \\
M & =1\end{aligned}$ & $2.73[1.4,4.0],<0.001^{c}$ \\
\hline $\begin{array}{l}\text { PROMIS Pain Interference } \\
\text { (range 0- }\end{array}$ & $3.38 \pm 5.0$ & $11.05 \pm 6.74$ & $7.67[4.3,11.1],<0.001$ \\
\hline PainDETECT & $3.86 \pm 4.21$ & $9.76 \pm 7.08$ & $5.90[2.1,9.7], 0.004$ \\
\hline Fibromyalgia survey criteria & $4.16 \pm 3.18$ & $8.26 \pm 4.39$ & $-4.10[-6.35,-1.86], 0.001$ \\
\hline Analgesic use ${ }^{a}$ & 27 (48\%) & $18(90 \%)$ & $0.10[0.02,0.49], 0.001$ \\
\hline $\begin{array}{l}\text { Nonpharmacologic pain } \\
\text { relieving methods used }^{\mathrm{b}}\end{array}$ & 24 (43\%) & 15 (75\%) & $0.25[0.08,0.78], 0.014$ \\
\hline $\begin{array}{l}\text { Median number of } \\
\text { healthcare visits in the } \\
\text { year }\end{array}$ & $5[\mathrm{IQR} 3,7]$ & $4[\mathrm{IQR} 3,6]$ & $P=0.358^{c}$ \\
\hline
\end{tabular}

Data are presented as mean \pm standard deviation or as $\mathrm{n}(\%)$, where applicable.

$\mathrm{Cl}$, Confidence Interval; M, Median; MD, Mean Difference; OR, Odds Ratio; PROMIS, PatientReported Outcome Measurement Information System.

${ }^{a}$ Reported analgesics were all over-the-counter nonopioids except for one in the Low Symptom group that used tramadol, one in the High Symptom group using oxycodone, and another in the High Symptom group using tizanidine.

bincludes heat/ice, meditation, acupressure, acupuncture, physical therapy, other.

${ }^{c} M a n n-W h i t n e y U$ test of significance.
TABLE 2 Description of the long-term outcomes in the cluster profile groups
TABLE 3 Associations between baseline characteristics and longterm pain interference scores based on the multivariable regression analysis

\begin{tabular}{|lcr|} 
& \multicolumn{1}{c}{$\boldsymbol{\beta}[95 \%$ Confidence } & \\
& Interval] & $\boldsymbol{P}$ value \\
\hline Age (years) & $0.39[-0.38,1.16]$ & 0.315 \\
\hline Body mass index $\left(\mathrm{kg} / \mathrm{m}^{2}\right)$ & $-0.17[-0.41,0.08]$ & 0.178 \\
\hline Sex (ref female) & $-0.53[-3.73,2.67]$ & 0.741 \\
\hline Spine levels fused & $-0.36[-1.10,0.38]$ & 0.332 \\
\hline $\begin{array}{l}\text { Intrathecal morphine used (ref other) } \\
\text { Hospital morphine consumption } \\
\text { (mg/kg/hr) }\end{array}$ & $0.47[-4.75,5.69]$ & 0.857 \\
\hline $\begin{array}{l}\text { Hospital average pain score (NRS } \\
\text { 0-10) }\end{array}$ & $0.29[-0.49,1.06]$ & 0.458 \\
\hline $\begin{array}{l}\text { Baseline Widespread Pain (ref None) } \\
\text { Symptom Cluster (ref Low Symptom }\end{array}$ & $-0.48[-3.37,2.40]$ & 0.739 \\
\hline \begin{tabular}{l} 
Cluster) \\
\hline
\end{tabular} & $9.92[6.63,13.2]$ & $<0.001$ \\
\hline
\end{tabular}

NRS, Numeric Rating Scale.

Clusters of symptoms that include depression, anxiety, and daytime fatigue have been described in children with chronic musculoskeletal and other pain conditions and have been correlated with poorer functioning. ${ }^{26,27}$ Higher levels of somatization, depression, and symptom internalization scores were found to predict persistent or recurrent pain trajectories in large community samples of children. ${ }^{28,29}$ These and other data suggest a "pain vulnerable" trait that emerges during childhood and that may reflect, in part, a neurobiological mechanism such as central pain sensitization or augmentation. ${ }^{29}$ Recent data correlating higher catecholamine levels in cerebrospinal fluid preoperatively with persistent pain 6 weeks after spine fusion suggest a potential underlying mechanism for pain sensitization. ${ }^{30}$ However, this study also found an association with elevated systemic catecholamine levels, and thus, a central mechanism for persistent pain remains hypothetical. Other reports ruled out physiologic or nociceptive causes for $65 \%$ of patients who reported persistent pain 23 years after adolescent spine fusion. ${ }^{31}$ Importantly, in the present study, fibromyalgia survey criteria scores did not contribute to cluster membership, but were significantly higher at baseline for children in the High Symptom Cluster (mean 10.93 vs 5.69 for the Low Symptom Cluster). These average scores in our high symptom group are lower than the reported total cutpoint for a diagnosis of fibromyalgia (ie, $\geq 13$ ); however, it has been suggested that central pain sensitization is a continuum, and that survey criteria scores provide a global conceptualization of this continuum. ${ }^{17}$ Whether higher pFSC scores indicate the early stages of pain sensitization is unclear from our findings. Further study that includes quantitative sensory testing in conjunction with our baseline measures is needed to better understand whether pain sensitization is a potential mechanism for persistent postoperative pain in children with scoliosis.

It is important to note that although we found ongoing pain outcome differences between the High and Low Symptom Clusters, children in the High Symptom Cluster did experience a decrease in their pain interference scores from baseline. This is somewhat similar to a recent longitudinal study demonstrating that among adolescents who reported higher pain at baseline, most reported improvements 1-5 years after surgery. ${ }^{7}$ This study also found that children whose pain improved had reported lower body image at baseline compared to children who experienced a worsening pain trajectory. The investigators suggested that improved overall body image following 
surgical correction of scoliosis may help to explain an improved longterm pain outcome.

The ability to generalize findings from this study is limited by the potential for symptom recall which could have biased the self-report of symptomology prior to surgery. However, we previously reported the stability of self-reported scores from the clinic visit to approximately 2 weeks later, supporting the reliability of recall in this age group. The contributions of various symptom measures to cluster membership differed slightly from our previous and smaller sample. The strong contributions of depression, catastrophizing, pain interference, and fatigue to cluster membership remained stable from our earlier study, whereas pFSC no longer helped to differentiate the children. However, the criterion validity of our resultant clusters was supported by the demonstrated differences between clusters in the theoretically related baseline variables, pain intensity and widespread pain. It will be important to examine whether similar symptom clusters can be differentiated and validated in larger and varied samples of children and whether the predictive value of a high symptom cluster remains important when controlled for other factors such as parental psychological measures. Lastly, $20 \%$ of our sample was lost to follow-up which could have biased our results. However, the characteristics of those children were similar to those who completed the 1-year follow-up, reducing the potential for nonresponse bias.

Findings from this exploratory study suggest that children with idiopathic scoliosis can be differentiated prior to undergoing posterior spine fusion by a cluster of symptomology that indicate vulnerability to a long-term pain trajectory. It is important to identify such pain vulnerable children before surgery so that a comprehensive plan of care might be initiated and individualized in a cost-efficient manner. This, in turn, could help to mitigate the potential negative impact that persistent postoperative pain can have on children's dayto-day physical and social functioning and quality of life.

\section{ACKNOWLEDGMENTS}

The authors thank the following persons who helped with subject recruitment, data collection, and data entry: Reilly Philliben, Caroline Hyman, and Marianna Dorta. No external funding was received for the support of this work; the authors report no conflicts of interest pertaining to this work.

\section{ETHICAL APPROVAL}

Approval was obtained from the Institutional Review Board at the University of Michigan (IRBMED), HUM00086972

\section{DISCLOSURES}

The authors have no conflicts of interest to report.

\section{ORCID}

Terri Voepel-Lewis iD http://orcid.org/0000-0001-9718-9695

\section{REFERENCES}

1. Fortier MA, Chou J, Maurer EL, et al. Acute to chronic postoperative pain in children: preliminary findings. J Pediatr Surg. 2011;46:17001705.

2. Page MG, Stinson J, Campbell F, et al. Identification of pain-related psychological risk factors for the development and maintenance of pediatric chronic postsurgical pain. J Pain Res. 2013;6:167-180.

3. Rabbitts JA, Fisher E, Rosenbloom BN, et al. Prevalence and predictors of chronic postsurgical pain in children: a systematic review and meta-analysis. J Pain. 2017;18:605-614.

4. Mossetti V, Boretsky $\mathrm{K}$, Astuto $\mathrm{M}$, et al. Persistent pain following common outpatient surgeries in children: a multicenter study in Italy. Paediatr Anaesth. 2018;28:231-236.

5. Connelly M, Fulmer RD, Prohaska J, et al. Predictors of postoperative pain trajectories in adolescent idiopathic scoliosis. Spine. 2014;39:E174-E181.

6. Perry M, Starkweather A, Baumbauer K, et al. Factors leading to persistent postsurgical pain in adolescents undergoing spinal fusion: an integrative literature review. J Pediatr Nurs. 2018;38:74-80.

7. Sieberg CB, Simons LE, Edelstein MR, et al. Pain prevalence and trajectories following pediatric spinal fusion surgery. J Pain. 2013;14:1694-1702.

8. Upasani VV, Caltoum C, Petcharaporn M, et al. Adolescent idiopathic scoliosis patients report increased pain at five years compared with two years after surgical treatment. Spine. 2008;33:1107-1112.

9. Bastrom TP, Marks MC, Yaszay B, et al. Prevalence of postoperative pain in adolescent idiopathic scoliosis and the association with preoperative pain. Spine. 2013;38:1848-1852.

10. Alpert HW, Farley FA, Caird MS, et al. Outcomes following removal of instrumentation after posterior spinal fusion. J Pediatr Orthop. 2014;34:613-617.

11. Rabbitts JA, Zhou C, Groenewald CB, et al. Trajectories of postsurgical pain in children: risk factors and impact of late pain recovery on long-term health outcomes after major surgery. Pain. 2015;156: 2383-2389.

12. Voepel-Lewis T, Caird MS, Tait AR, et al. A high preoperative pain and symptom profile predicts worse pain outcomes for children after spine fusion surgery. Anesth Analg. 2017;124:1594-1602.

13. Brummett CM, Urquhart AG, Hassett AL, et al. Characteristics of fibromyalgia independently predict poorer long-term analgesic outcomes following total knee and hip arthroplasty. Arthritis Rheumatol. 2015;67:1386-1394.

14. Voepel-Lewis T, Burke CN, Jeffreys N, et al. Do 0-10 numeric rating scores translate into clinically meaningful pain measures for children? Anesth Analg. 2011;112:415-421.

15. Zernikow B, Gerhold K, Burk G, , et al. [Definition, diagnosis and therapy of chronic widespread pain and so-called fibromyalgia syndrome in children and adolescents. Systematic literature review and guideline]. Schmerz. 2012;26:318-330.

16. Ting TV, Barnett K, Lynch-Jordan A, et al. 2010 American college of rheumatology adult fibromyalgia criteria for use in an adolescent female population with juvenile fibromyalgia. J Pediatr. 2016;169 (181-187):e181.

17. Clauw DJ. Fibromyalgia: a clinical review. JAMA. 2014;311:1547-1555.

18. Rabbitts JA, Holley AL, Groenewald CB, et al. Association between widespread pain scores and functional impairment and health-related quality of life in clinical samples of children. J Pain. 2016;17(6):678-684.

19. Irwin DE, Gross HE, Stucky BD, et al. Development of six PROMIS pediatrics proxy-report item banks. Health Qual Life Outcomes. 2012;10:22.

20. Varni JW, Magnus B, Stucky BD, et al. Psychometric properties of the PROMIS (R) pediatric scales: precision, stability, and comparison of different scoring and administration options. Qual Life Res. 2014;23:1233-1243. 
21. Vervoort T, Goubert L, Eccleston C, et al. Catastrophic thinking about pain is independently associated with pain severity, disability, and somatic complaints in school children and children with chronic pain. J Pediatr Psychol. 2006;31:674-683.

22. Sambandam R. Collinearity is a natural problem in clustering. Marketing Res. 2003;15:16-21.

23. Mooi E, Sarstedt M (eds). Cluster Analysis. In: A concise guide to market research. Berlin, Germany: Springer-Verlag; 2011:978-3.

24. Chidambaran V, Ding L, Moore DL, et al. Predicting the pain continuum after adolescent idiopathic scoliosis surgery: a prospective cohort study. Eur J Pain. 2017;21:1252-1265.

25. Birnie KA, Chorney J, El-Hawary R, et al. Child and parent pain catastrophizing and pain from presurgery to 6 weeks postsurgery: examination of cross-sectional and longitudinal actor-partner effects. Pain. 2017;158:1886-1892.

26. Hoftun GB, Romundstad PR, Rygg M. Factors associated with adolescent chronic non-specific pain, chronic multisite pain, and chronic pain with high disability: the Young-HUNT Study 2008. J Pain. 2012;13:874-883.

27. King $S$, Chambers $C T$, Huguet $A$, et al. The epidemiology of chronic pain in children and adolescents revisited: a systematic review. Pain. 2011;152:2729-2738.
28. Dunn KM, Jordan KP, Mancl L, et al. Trajectories of pain in adolescents: a prospective cohort study. Pain. 2011;152:66-73.

29. Kroner-Herwig B, Gassmann J, van Gessel H, et al. Multiple pains in children and adolescents: a risk factor analysis in a longitudinal study. J Pediatr Psychol. 2011;36:420-432.

30. Ferland CE, Parent AJ, Saran N, et al. Preoperative norepinephrine levels in cerebrospinal fluid and plasma correlate with pain intensity after pediatric spine surgery. Spine Deform. 2017;5: 325-333.

31. Danielsson AJ, Nachemson AL. Back pain and function 23 years after fusion for adolescent idiopathic scoliosis: a case-control studypart II. Spine. 2003;28:E373-E383.

How to cite this article: Voepel-Lewis T, Caird MS, Tait AR, et al. A cluster of high psychological and somatic symptoms in children with idiopathic scoliosis predicts persistent pain and analgesic use 1 year after spine fusion. Pediatr Anesth. 2018;28:873-880. https://doi.org/10.1111/pan.13467 